\title{
Phase II trial of carboplatin and bevacizumab in patients with breast cancer brain metastases
}

Jose Pablo Leone ${ }^{1}$, Kyrre E. Emblem², Michelle Weitz ${ }^{1}$, Rebecca S. Gelman', Bryan P. Schneider ${ }^{3}$, Rachel A. Freedman ${ }^{1}$, Jerry Younger ${ }^{4}$, Marco C. Pinho ${ }^{5}$, A. Gregory Sorensen 6 , Elizabeth R. Gerstner ${ }^{4}$, Gordon Harris ${ }^{4}$, lan E. Krop ${ }^{1}$, Daniel Morganstern' ${ }^{1}$, Jessica Sohl ${ }^{1}$, Jiani Hu', Elizabeth Kasparian ${ }^{1}$, Eric P. Winer ${ }^{1}$ and Nancy U. $\operatorname{Lin}^{1 *}$ (D)

\begin{abstract}
Background: We aimed to examine the safety and efficacy of bevacizumab and carboplatin in patients with breast cancer brain metastases.

Methods: We enrolled patients with breast cancer and $\geq 1$ measurable new or progressive brain metastasis. Patients received bevacizumab $15 \mathrm{mg} / \mathrm{kg}$ intravenously (IV) on cycle 1 day 1 and carboplatin IV AUC =5 on cycle 1 day 8. Patients with HER2-positive disease also received trastuzumab. In subsequent cycles, all drugs were administered on day 1 of each cycle. Contrast-enhanced brain MRI was performed at baseline, 24-96 $\mathrm{h}$ after the first bevacizumab dose (day +1$)$, and every 2 cycles. The primary endpoint was objective response rate in the central nervous system (CNS ORR) by composite criteria. Associations between germline VEGF single nucleotide polymorphisms (rs699947, rs2019063, rs1570360, rs833061) and progression-free survival (PFS) and overall survival (OS) were explored, as were associations between early (day +1$)$ MRI changes and outcomes.

Results: Thirty-eight patients were enrolled (29 HER2-positive, 9 HER2-negative); all were evaluable for response. The CNS ORR was 63\% (95\% Cl, 46-78). Median PFS was 5.62 months and median OS was 14.10 months. As compared with an Eastern Cooperative Oncology Group performance status (ECOG PS) of 0, patients with ECOG PS $1-2$ had significantly worse PFS and OS (all $P<0.01$ ). No significant associations between VEGF genotypes or early MRI changes and clinical outcomes were observed.
\end{abstract}

Conclusions: The combination of bevacizumab and carboplatin results in a high rate of durable objective response in patients with brain metastases from breast cancer. This regimen warrants further investigation.

Trial registration: NCT01004172. Registered 28 October 2009.

Keywords: Breast cancer, Brain metastases, Bevacizumab

\footnotetext{
* Correspondence: nlin@partners.org

'Dana-Farber Cancer Institute, Dana-Farber/Brigham \& Women's Cancer Center, 450 Brookline Avenue, Boston, MA 02215, USA

Full list of author information is available at the end of the article
}

(c) The Author(s). 2020 Open Access This article is licensed under a Creative Commons Attribution 4.0 International License, which permits use, sharing, adaptation, distribution and reproduction in any medium or format, as long as you give appropriate credit to the original author(s) and the source, provide a link to the Creative Commons licence, and indicate if changes were made. The images or other third party material in this article are included in the article's Creative Commons licence, unless indicated otherwise in a credit line to the material. If material is not included in the article's Creative Commons licence and your intended use is not permitted by statutory regulation or exceeds the permitted use, you will need to obtain permission directly from the copyright holder. To view a copy of this licence, visit http://creativecommons.org/licenses/by/4.0/. The Creative Commons Public Domain Dedication waiver (http://creativecommons.org/publicdomain/zero/1.0/) applies to the data made available in this article, unless otherwise stated in a credit line to the data. 


\section{Introduction}

Up to half of patients with human epidermal growth factor receptor 2 (HER2)-positive or triple-negative metastatic breast cancer, and $10-15 \%$ of patients with estrogen receptor (ER)-positive/HER2-negative metastatic breast cancer, will develop brain metastases [1, 2]. The management of patients with brain metastases is a continuing challenge.

Brain metastases from breast cancer are highly vascular and exhibit abnormal vessel morphology [3]. Preclinical data suggest that vascular endothelial growth factor (VEGF) promotes the growth of breast cancer brain metastases and that treatment with antiangiogenic agents results in tumor regression $[4,5]$. Bevacizumab is a humanized, monoclonal antibody directed against VEGF. At the time the study was designed, improvements in the objective response rate (ORR) and progression-free survival (PFS) had been reported among patients with HER2-negative metastatic breast cancer when bevacizumab was added to chemotherapy, and dual antibody blockade with bevacizumab and trastuzumab was shown to produce objective responses in HER2-positive disease $[6,7]$. Evidence from autopsy series indicates that platinum agents reach therapeutic concentrations in brain metastases [8]. The combination of cisplatin and etoposide has been reported in a small study to lead to a central nervous system (CNS) ORR of $38 \%$ in breast cancer patients, albeit in a patient population that was less refractory (no prior cranial radiotherapy, more than two thirds of patients chemotherapy-naive) than typical contemporary patients [9]. In addition, preclinical data suggest a possible synergism between bevacizumab and carboplatin in glioma models [10].

Since this study was initiated, multiple randomized studies have failed to demonstrate an overall survival benefit for bevacizumab in metastatic breast cancer and regulatory approval in the USA was withdrawn [11-15]. However, patients with active brain metastases were excluded due to concerns about intracranial hemorrhage. Thus, whether brain metastases may be particularly sensitive to angiogenic blockade remains an understudied question.

In this study, we evaluated the safety and efficacy of bevacizumab and carboplatin in breast cancer patients with new or progressive brain metastases. We also explored several potential predictors of outcome, including VEGF genotype and early imaging changes.

\section{Patients and methods}

\section{Patients}

We enrolled 38 patients with metastatic breast cancer and measurable CNS disease ( $\geq 1$ parenchymal brain lesion with longest diameter $>10 \mathrm{~mm}$ ), new or progressive CNS lesion(s), Eastern Cooperative Oncology Group performance status (ECOG PS) 0-2, no increase in corticosteroid dose in the week prior to the baseline brain MRI, left ventricular ejection fraction $\geq 50 \%$, and adequate organ function. Patients could have received prior carboplatin if not given with bevacizumab. Prior bevacizumab was allowed if it had not been given within 6 months prior to the diagnosis of CNS metastases. Prior whole brain radiation therapy (WBRT) and/or stereotactic radiosurgery (SRS) was allowed but not required. For patients who had received prior radiation therapy (RT), patients had to have developed subsequent CNS progression or the presence of residual untreated lesion(s) (which would be followed as target lesion[s]). There was no limit on the number of prior therapies. Key exclusion criteria were contraindication to MRI, leptomeningeal carcinomatosis as the only site of CNS involvement, and more than 2 seizures over the last 4 weeks prior to study entry. Bevacizumab-specific exclusions included uncontrolled hypertension, history of stroke or myocardial infarction within 6 months, New York Heart Association (NYHA) Class II or higher congestive heart failure, evidence of bleeding diathesis, need for full-dose anticoagulation, or major surgical procedure within 28 days.

\section{Study design}

This was an open-label, nonrandomized, phase II study. Patients were enrolled into two simultaneously enrolling cohorts: HER2-negative and HER2-positive. In order to support the correlative MRI imaging endpoint, all patients received bevacizumab monotherapy $15 \mathrm{mg} / \mathrm{kg}$ intravenously on cycle 1 day 1 (C1D1). On C1D8, HER2-negative patients received carboplatin IV AUC = 5. HER2-positive patients also received trastuzumab (8 $\mathrm{mg} / \mathrm{kg}$ load if indicated; otherwise, $6 \mathrm{mg} / \mathrm{kg}$ ) on C1D8. Three weeks later, patients received both carboplatin $(\mathrm{AUC}=5)$ and bevacizumab $(15 \mathrm{mg} / \mathrm{kg})$ on day 1 (plus trastuzumab $6 \mathrm{mg} / \mathrm{kg}$, if HER2-positive) and every 3 weeks thereafter.

No dose modifications were allowed for bevacizumab or trastuzumab, which could be held in case of adverse events. For carboplatin, the protocol-specified dose holds and reductions were based upon both the absolute neutrophil count (ANC) and platelet count, as well as for grade 3/4 non-hematological toxicities. If one (or more) drug is needed to be discontinued, the patient could remain on protocol and the other drug(s) continued. Patients continued protocol therapy until disease progression in CNS and/or extracranial sites, unacceptable adverse events (AEs), physician's judgment, or withdrawal of consent.

The protocol was approved by the Dana-Farber/Harvard Cancer Center (DF/HCC) Scientific Review Committee and Institutional Review Board. The study is registered at www.clinicaltrials.gov (NCT01004172). All 
study participants provided written informed consent prior to any study-related procedures.

\section{Study assessments}

Patients were evaluated at baseline, C1D8, and day 1 of all subsequent cycles. A neurological examination worksheet was completed by the treating provider at each study visit. AEs were assessed according to the National Cancer Institute Common Terminology Criteria for Adverse Events version 3.0. Non-CNS restaging scans (CT or MRI) and brain MRI sequences were obtained at baseline and after every 2 cycles. If non-CNS disease remained stable after 4 cycles, the frequency of non-CNS imaging was decreased to once every 4 cycles. Left ventricular ejection fraction (LVEF) was assessed every 4 cycles.

The primary efficacy endpoint was CNS ORR, according to composite criteria. Protocol pre-specified response evaluation for this study was done using RECIST 1.0 for non-CNS lesions and composite criteria (which incorporated centrally assessed volumetric response) for CNS lesions according to previously published methods [16]. The MRI series used for response assessment of CNS lesions was T1-weighted gadolinium-enhanced. For imaging at all timepoints, MRI minimum requirements were to have $5 \mathrm{~mm}$ or less slice thickness for axial post-contrast images using T1 or SPGR sequence. Of note, all MRI scans at baseline, following the first dose of bevacizumab and prior to cycle 3, were performed centrally on the same machines at Massachusetts General Hospital Charlestown Navy Yard according to the following specifications, which included voxel size $0.5 \times 0.5 \times 0.8$ (Supplemental Figure 1). All brain MRI scans were reviewed centrally by the DF/HCC Tumor Imaging Metrics Core. CNS partial response required all of the following: $>50 \%$ reduction in volumetric sum of all measurable brain metastases compared to baseline, no progression of non-measurable lesions, no new lesions, stable or decreasing steroid dose, no new/progressive tumor-related neurologic signs or symptoms, and no progression of non-CNS disease by RECIST 1.0. CNS complete response required the same items as partial response but with complete resolution of all measurable and non-measurable brain metastases. $\mathrm{Pa}$ tients were considered to have progressive disease if any of the following occurred: $>40 \%$ increase in the volumetric sum of all measurable lesions as compared to nadir, progression of non-measurable lesions, new lesion(s), increasing steroid requirement, new or progression tumor-related neurologic signs and symptoms, or progression of non-CNS disease by RECIST 1.0. Secondary clinical endpoints included safety/tolerability, PFS, clinical benefit rate (complete response + partial response + stable disease $\geq 24$ weeks), site of first progression, and overall survival (OS).

\section{Correlative studies}

Blood for VEGF genotyping of four pre-specified VEGF single nucleotide polymorphisms (rs699947, rs2019063, rs1570360, rs833061) was collected at baseline. Associations between VEGF genotyping and PFS and OS were evaluated by log-rank tests.

MRI of the brain was performed on a 3 Tesla Siemens TrioTim, including conventional T2-weighted, fluidattenuated inversion recovery (FLAIR) and T1-weighted imaging (with and without gadolinium). Imaging was performed at baseline, 24-96 $\mathrm{h}$ after C1D1 bevacizumab $($ day +1$)$, and after 2 cycles of treatment. Thirty-eight patients had evaluable MRIs. Contrast-enhancing lesions and areas of T2 abnormality on FLAIR images were outlined by an experienced neuroradiologist blinded to patient identity and time of visit by using a semi-automatic volumetric approach in $3 D$ Slicer [17]. The total number of image voxels formed the basis for the overall lesion volume.

\section{Statistical analysis}

The HER2-positive and HER2-negative cohorts were analyzed together. All patients who received at least one dose of protocol therapy were included in the toxicity analysis. The analysis of objective response was limited to patients who received at least one dose of study treatment and who had measurable CNS disease, defined as at least one CNS lesion $>10 \mathrm{~mm}$ in longest dimension, confirmed by central radiology review. The study was designed to distinguish between a CNS response rate of $5 \%$ versus $20 \%$, with responses in at least 1 of 12 assessable patients entered in the first stage in order to proceed to full accrual. At the end of the study, if responses were observed in $>4$ of 37 assessable patients, the regimen would be declared worthy of further study. The study included a stopping rule for CNS hemorrhage. If 3 or more patients experienced a grade 2 or higher CNS bleeding event, the study would be terminated. With this design, there was a $73 \%$ chance of terminating the study if the true probability of CNS hemorrhage is $10 \%$, and a $93 \%$ chance of early termination if the true probability is $15 \%$.

Initially, the study was designed to accrue up to 20 HER2-positive and up to 20 HER2-negative patients, in order to explore the CNS response rate according to HER2 status. However, because of slow accrual to the HER2-negative cohort, the study was amended on August 23, 2011, to remove the limit on the number of maximum patients per cohort and to remove formal analyses of clinical outcomes by HER2 status. 
PFS was defined as the time from start of treatment to time of progression, second cancer, or death, whichever occurred first. Patients who were alive and progressionfree at the date of last contact were censored at the date of last contact. OS was defined as the time from start of treatment until death. Patients who were alive at the date of last contact were censored on that date. PFS and OS were estimated by Cox proportional hazards regression models with hazard ratios (HR) with 95\% confidence intervals $(\mathrm{CI})$ and presented by Kaplan-Meier plots. Significance level was $P<0.05$ with HolmBonferroni corrections for multiple comparisons.

\section{Results}

\section{Patients and treatment}

Thirty-eight women were enrolled between November 2009 and August 2012 (29 HER2-positive, 9 HER2negative). As shown in Table $1,87 \%$ of patients were ECOG PS $0-1$ on study entry, and patients had received a median of 3 lines of chemotherapy in the metastatic setting (range 0-10). Most patients $(33 / 38 ; 87 \%)$ had previously received WBRT, SRS, or both, with median time from last radiation to study entry of 7.5 months. Among patients with HER2-positive disease, all but one had prior trastuzumab and $70 \%$ had prior lapatinib. Patients had to be on stable or decreasing doses of corticosteroids for at least 7 days prior to enrollment. Investigator-rated neurological signs and symptoms were collected throughout the study (Supplemental Figure 2). Half of patients were denoted as having at least one sign or symptom at study baseline. Seven patients (18.4\%) were taking corticosteroids at baseline.

At time of data-lock (June 1, 2017), no patients were still receiving protocol therapy. The reasons for protocol therapy discontinuation were progression in CNS only $(n=16)$, progression in non-CNS only $(n=16)$, progression in both CNS and non-CNS $(n=2)$, treatmentrelated toxicity $(n=1)$, physician or patient decision $(n=$ $1)$, and symptomatic deterioration $(n=2)$.

\section{Efficacy}

As shown in Table 2, according to protocol pre-specified composite criteria for response, the CNS ORR in the 38 assessable patients was $63 \%$ (95\% CI, 47-77\%). Responses were seen in both HER2-positive and HER2negative patients. In a post hoc analysis, we also calculated the CNS ORR by response assessment in neurooncology brain metastases (RANO-BM) criteria and noted a CNS ORR of $47 \%$ [18]. Of the 27 patients with measurable non-CNS disease at baseline, objective nonCNS responses were observed in $31 \%$ of patients overall, but at a higher frequency in HER2-positive patients. Median PFS (which included both CNS and non-CNS progression events) was 5.62 months (95\% CI, 5.03-6.51 months) (Fig. 1a). At the time of the data-lock, all patients had died. Median OS was 14.1 months (95\% CI, 11.7-20.7 months) (Fig. 1b). Figure 2 shows the MRI of a patient at baseline and after 10 months of protocol therapy, showing partial response of her left occipital lesion from $2.99 \mathrm{cc}(16.4 \mathrm{~mm})$ at baseline to $0.59 \mathrm{cc}(9.1$ $\mathrm{mm})$.

\section{Safety}

The most commonly reported AEs are listed in Table 3. Overall, the regimen was well tolerated. A total of 272 cycles of bevacizumab were administered, with a median of 8 cycles (range 1-20) per patient. The most common reason for dose delay or hold was hematological toxicity (thrombocytopenia, neutropenia without fever), which was most likely due to the carboplatin. Three cases of grade $1 \mathrm{CNS}$ hemorrhage and one case of grade $2 \mathrm{CNS}$ hemorrhage were reported (Supplemental Table 1). There were no cases of grade 3 or 4 CNS hemorrhage. Carboplatin dose reductions were required in 23 patients. The most common reason for carboplatin dose reduction was thrombocytopenia $(n=22)$. Cardiac function was assessed every 4 cycles on study. Asymptomatic declines in LVEF < 50\% were observed in 5 (13\%) patients, with nadir ranging from 44 to $48 \%$.

\section{VEGF genotyping}

DNA was extracted from 29 blood samples. No significant association was found between VEGF genotypes and PFS or OS (Supplemental Table 2).

\section{Early MRI findings}

A brain MRI was performed between 24 and $96 \mathrm{~h}$ after C1D1 of bevacizumab (day +1$)$. The goal of this analysis was to assess whether changes from baseline volume to volume at day +1 would be associated with PFS and OS. To explore this association, we divided the patient cohort by the median into two groups that were compared: a group who had below the median decrease in volume of contrast-enhancing lesions on day +1 , and a group who had above the median decrease in volume of contrast-enhancing lesions on day +1 .

Figure 3 shows the Kaplan-Meier curves for PFS by the degree of decrease in volume between baseline MRI and day +1 MRI. There were no significant differences in PFS between patients who had below the median decrease in volume and those who had above the median decrease in volume, both when absolute decrease in volume was measured $(P=0.77$; Fig. $3 a)$ as well as when relative decrease in volume was measured $(P=0.88$; Fig. 3b).

We observed similar findings for OS (Fig. 4). Patients who had below the median decrease in volume had no significant differences in OS compared with those who 
Table 1 Patient and tumor characteristics

Characteristic

Age, years
Median (range)

Sex

$$
\begin{aligned}
& \text { Female } \\
& \text { Male }
\end{aligned}
$$

Race

White

Black or African-American

Asian

\section{Ethnicity}

$$
\text { Hispanic or Latino }
$$

\section{ECOG PS at baseline}

$$
0
$$$$
1
$$$$
2
$$

Stage at initial diagnosis

$$
\text { । }
$$

\section{Receptor status at initial diagnosis}

$$
\begin{aligned}
& \text { ER and/or PR+, HER2-negative } \\
& \text { ER and/or PR+, HER2-positive } \\
& \text { ER and PR negative, HER2-positive } \\
& \text { ER, PR, and HER2-negative }
\end{aligned}
$$

\section{Disease-free interval}

$$
\begin{aligned}
& \leq 2 \text { years } \\
& >2 \text { years }
\end{aligned}
$$

Interval from metastatic diagnosis to CNS diagnosis (median [months], range)

Interval from CNS diagnosis to study entry (median [months], range)

Number of metastatic disease sites (median, range)

\section{Sites of disease}

$$
\begin{aligned}
& \text { CNS } \\
& \text { Lung or pleural effusion } \\
& \text { Liver } \\
& \text { Bone } \\
& \text { Breast or chest wall } \\
& \text { Other }
\end{aligned}
$$

$\begin{array}{lll}\text { All }(n=38) & \begin{array}{l}\text { Cohort } 1 \text { (HER2-negative) } \\ (n=9)\end{array} & \begin{array}{l}\text { Cohort } 2 \text { (HER2-positive) } \\ (n=29)\end{array}\end{array}$

No. of patients (\%) No. of patients (\%) No. of patients (\%)

$48(31-62) \quad 46(31-57) \quad 48(32-62)$

$\begin{array}{lll}38(100) & 9(100) & 29(100) \\ 0(0) & 0(0) & 0(0)\end{array}$

$34(89) \quad 8(89)$

$1(3) \quad 1(11) \quad 0(0)$

$3(8) \quad 3(0) \quad 3(10)$

$1(3) \quad 0(0)$

$37(97) \quad 9(100) \quad 28(97)$

$24(63) \quad 4(44) \quad 20(69)$

$9(24) \quad 3(33) \quad 6(21)$

$5(13) \quad 2(22) \quad 3(10)$

$\begin{array}{lll}5(13) & 1(11) & 4(14) \\ 13(34) & 4(44) & 9(31) \\ 7(18) & 1(11) & 6(21) \\ 13(34) & 3(33) & 10(34)\end{array}$

$7(18) \quad 5(56)$

$14(37) \quad 1(11)^{\mathrm{a}} \quad 13(45)$

14(37) $0(0) \quad 14(48)$

$3(8) \quad 3(33) \quad 0(0)$

$\begin{array}{lll}22(58) & 7(78) & 15(52) \\ 16(42) & 2(22) & 14(48) \\ 14.8(0-107) & 5.9(0-44.7) & 16.6(0-107) \\ 15.7(0.2-63.6) & 11.0(0.4-25.5) & 19.8(0.2-63.6) \\ 4(1-5) & 4(1-5) & 4(1-5) \\ & & \\ 38(100) & 9(100) & 29(100) \\ 19(50) & 6(67) & 13(45) \\ 17(45) & 4(44) & 13(45) \\ 26(68) & 7(78) & 19(66) \\ 5(13) & 1(11) & 4(14) \\ 15(39) & 4(44) & 11(38) \\ \text { Median (range) } & \text { Median (range) } & \text { Median (range) } \\ 5.3(0.7,58.8) & 8.2(2.1,24.7) & 3.6(0.7,58.8)\end{array}$


Table 1 Patient and tumor characteristics (Continued)

\begin{tabular}{llll}
\hline Characteristic & All $(\boldsymbol{n}=\mathbf{3 8})$ & $\begin{array}{l}\text { Cohort } \mathbf{1} \text { (HER2-negative) } \\
(\boldsymbol{n}=\mathbf{9})\end{array}$ & $\begin{array}{l}\text { Cohort 2 (HER2-positive) } \\
(\boldsymbol{n}=\mathbf{2 9})\end{array}$ \\
& No. of patients (\%) & No. of patients (\%) & No. of patients (\%) \\
\hline Number of target CNS lesions & No. of patients (\%) & No. of patients (\%) & $7(24)$ \\
1 & & & $12(41)$ \\
2 & $9(24)$ & $2(22)$ & $4(14)$ \\
3 & $14(37)$ & $3(33)$ & $5(17)$ \\
4 & $7(18)$ & $1(11)$ & $1(3)$ \\
5 & $6(16)$ & $1(11)$ & Median (range) \\
Size of target CNS lesions (in $\mathbf{m m})$ & $2(5)$ & Median (range) & $14.4(10,60.5)$
\end{tabular}

${ }^{a}$ One patient with ER-positive, HER2-positive breast cancer was entered in cohort 1 because of prior intolerance to trastuzumab and was treated with carboplatin and bevacizumab (without trastuzumab)

${ }^{\text {b} T w o ~ p a t i e n t s ~ h a d ~ E R-p o s i t i v e, ~ H E R 2-n e g a t i v e ~ b r e a s t ~ c a n c e r ~ a t ~ d i a g n o s i s ~ b u t ~ s u b s e q u e n t ~ b i o p s y ~ a t ~ r e c u r r e n c e ~ s h o w e d ~ H E R 2-p o s i t i v e ~ d i s e a s e ~}$

Abbreviations: CNS central nervous system, ECOG PS Eastern Cooperative Oncology Group Performance Status, ER estrogen receptor, HER2 human epidermal growth factor receptor $2, P R$ progesterone receptor

had above the median decrease in volume, both in terms of absolute decrease in volume $(P=0.52$; Fig. $4 \mathrm{a})$ as well as relative decrease in volume $(P=0.73$; Fig. $4 \mathrm{~b})$.

We subsequently conducted landmark analyses for PFS and OS at the first follow-up MRI and at the second follow-up MRI. There were no significant associations between the degree of volume decrease by the first follow-up MRI and PFS or OS, nor between the degree of volume decrease by the second follow-up MRI and PFS or OS (data not shown).

The only variable with significant associations with PFS and OS was ECOG PS at baseline. As compared with ECOG PS $=0$, patients with ECOG PS $=1(\mathrm{HR}=$ $4.65 ; P=0.001)$ and ECOG PS $=2(\mathrm{HR}=9.71 ; P<0.001)$ had significantly worse PFS. Similarly, as compared with ECOG $\mathrm{PS}=0$, patients with ECOG $\mathrm{PS}=1 \quad(\mathrm{HR}=5.37$; $P=0.001)$ and ECOG PS $=2(\mathrm{HR}=18.67 ; P<0.001)$ experienced significantly worse OS.

\section{Discussion}

We evaluated the safety and efficacy of carboplatin and bevacizumab in patients with new or progressive breast cancer brain metastases. We found that the regimen was generally well tolerated and associated with a CNS ORR of $63 \%$ (95\% CI $47-77$ ) by pre-specified volumetric criteria, with responses seen across breast cancer subtypes. CNS ORR calculated retrospectively using RANO-BM criteria was $47 \%$.

Since our study was initiated, the role of bevacizumab in metastatic breast cancer has undergone a major evolution, and its U.S. regulatory approval was revoked in November 2011 [11, 13, 14, 19-21]. Despite this, it is important to note that none of the phase III trials included patients with active brain metastases. We believe our results support further evaluation of bevacizumab in the treatment of patients with breast cancer brain metastases. Our efficacy results are consistent with data from

Table 2 Summary table of CNS best response by composite criteria among evaluable patients

\begin{tabular}{llll}
\hline Response & All $(\boldsymbol{n}=\mathbf{3 8})$ & Cohort $\mathbf{1}$ (HER2-negative) $(\boldsymbol{n}=\mathbf{9})$ & $\begin{array}{l}\text { Cohort 2 (HER2-positive) }(\boldsymbol{n}=\mathbf{2 9}) \\
\text { No. of patients (\%) }\end{array}$ \\
\hline No. of patients (\%) & No. of patients (\%) & $0(0)$ \\
PR & $0(0)$ & $0(0)$ & $19(66)$ \\
SD $\geq 24$ weeks & $24(63)^{\mathrm{a}}$ & $5(56)$ & $2(7)$ \\
SD $<24$ weeks & $2(5)$ & $0(0)$ & $5(17)$ \\
PD & $5(13)$ & $0(0)$ & $3(10)$ \\
PD in CNS by radiographic criteria & $7(18)$ & $4(44)$ & $1(3)$ \\
Symptomatic deterioration & $1(3)$ & $0(0)$ & $1(3)$ \\
PD in non-CNS site(s) & $2(5)$ & $1(11)$ & $1(3)$ \\
\hline
\end{tabular}

${ }^{a}$ Taking into account the two-stage design, the $95 \% \mathrm{Cl}$ for CNS ORR $(n=38)$ is 47 to $77 \%$

${ }^{b}$ Patients were considered to have progressed if any of the PD criteria were met. Each patient could also have been taken off study for meeting multiple PD criteria simultaneously

Abbreviations: CNS central nervous system, $C R$ complete response, HER2 human epidermal growth factor receptor 2, $P D$ progression of disease, $P R$ partial response, $S D$ stable disease 

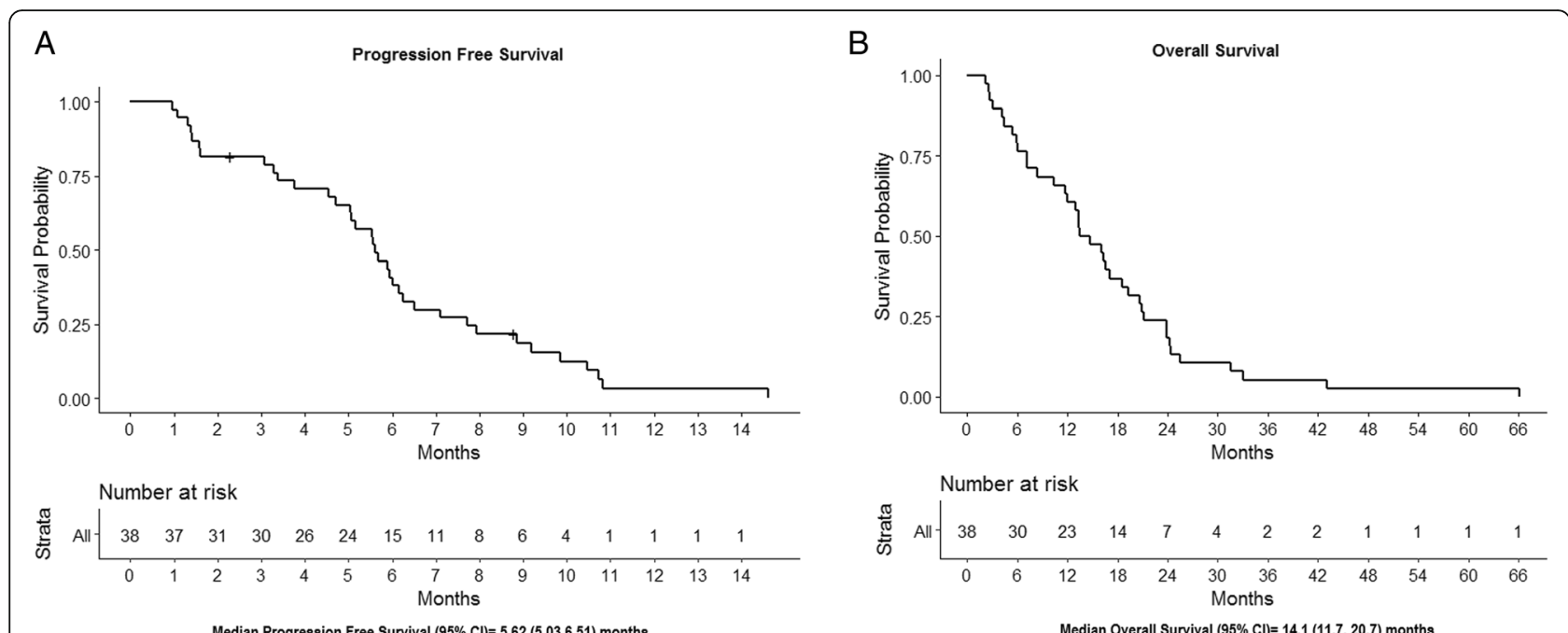

Fig. 1 Progression-free survival (a) and overall survival (b) Kaplan-Meier plot. Two patients came off-treatment before disease progression. They were censored at the off-treatment time point
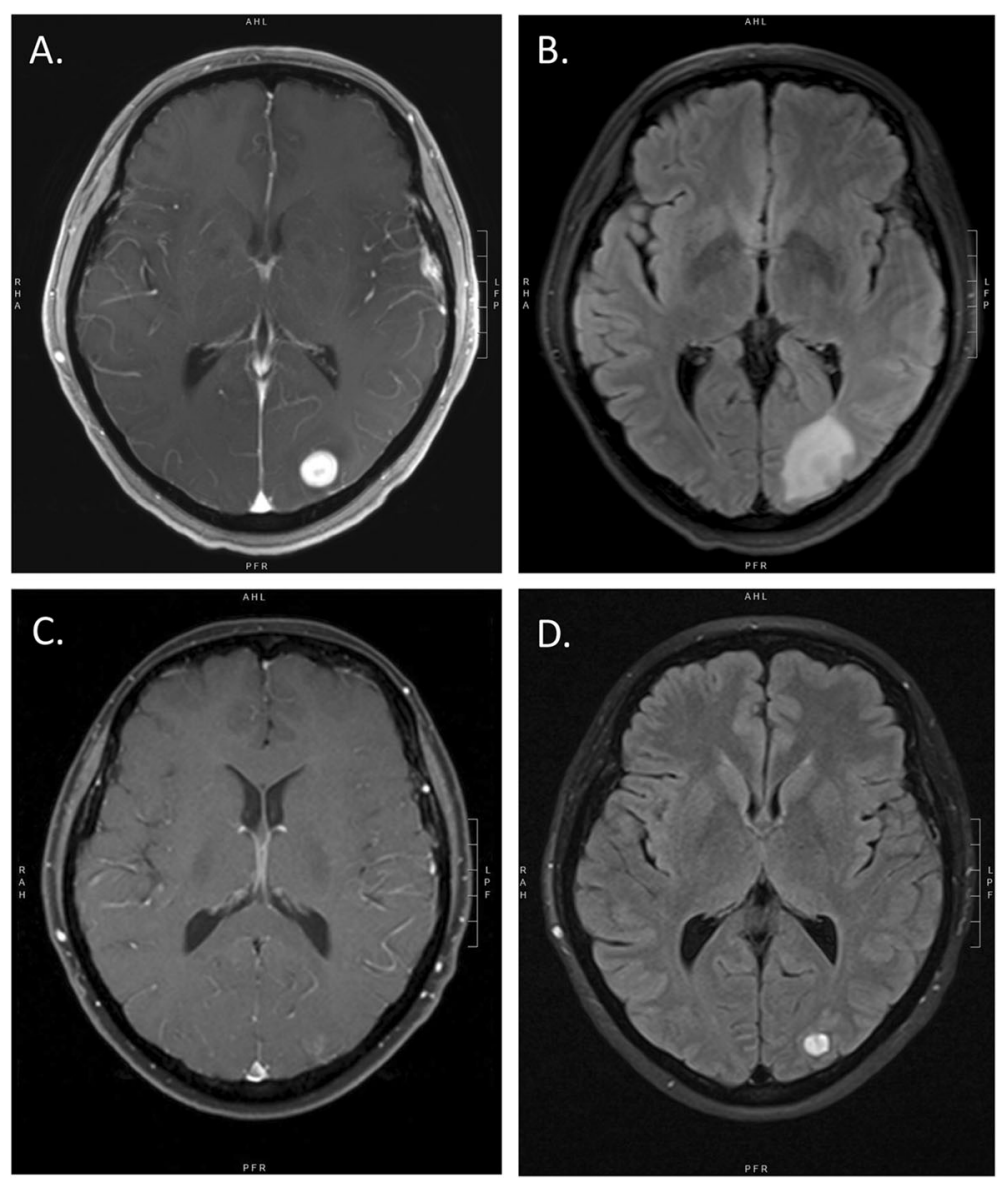

Fig. 2 Brain MRI of a representative study participant at baseline, showing axial, T1-weighted, post-gadolinium (a) and T2-FLAIR (b) images. Brain MRI of the same participant after 10 months of protocol therapy, showing axial, T1-weighted, post-gadolinium (c) and T2-FLAIR (d) images, consistent with response to treatment 
Table 3 Summary of adverse events related to treatment with at least 10\% incidence-by cohort

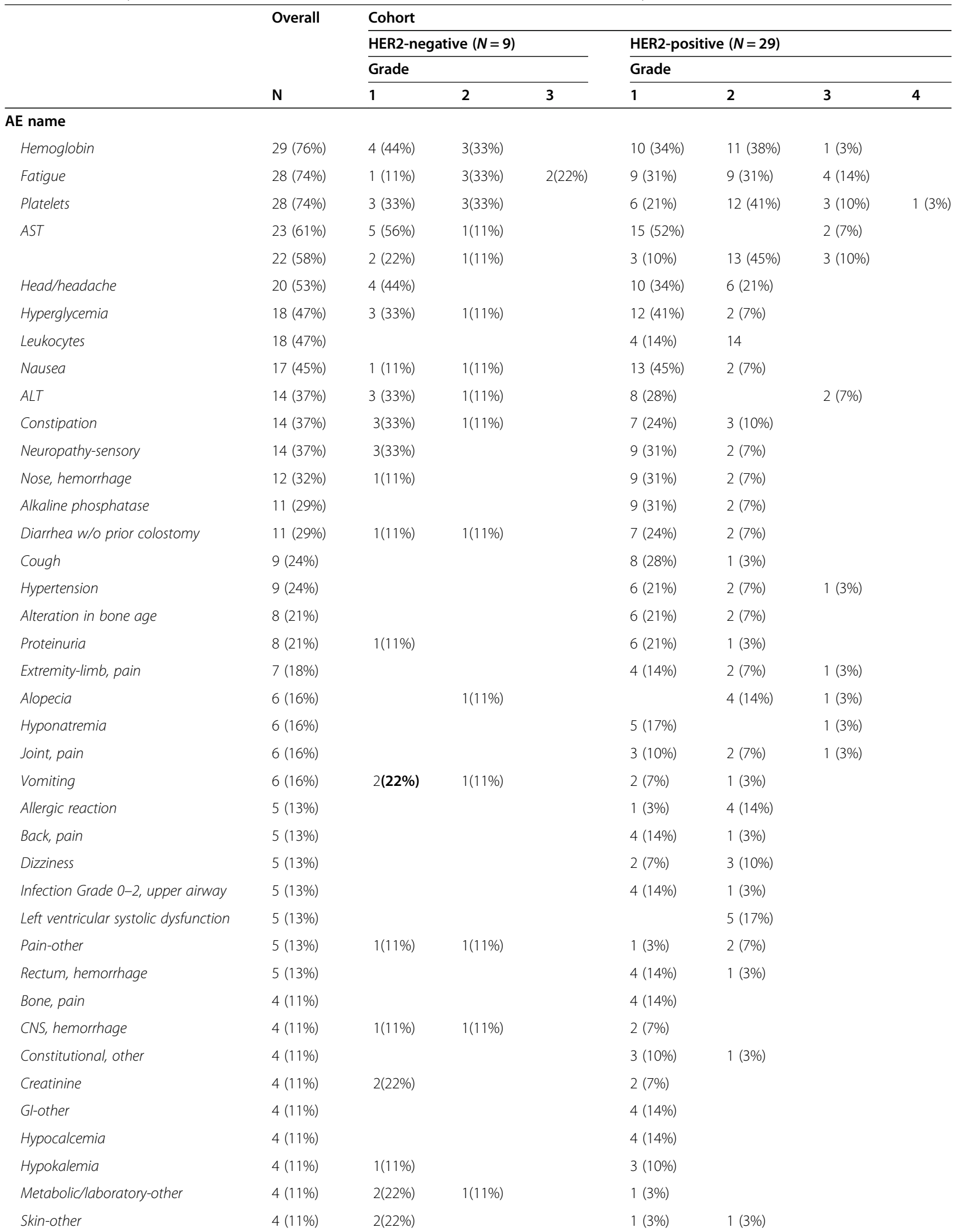


Table 3 Summary of adverse events related to treatment with at least 10\% incidence-by cohort (Continued)

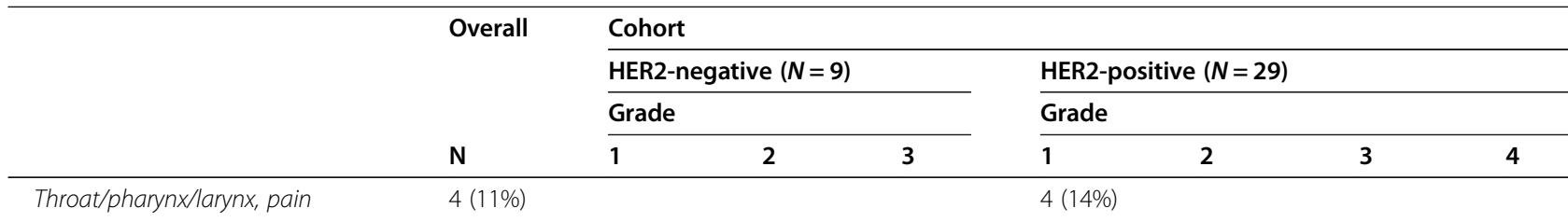

Abbreviations: AE adverse event, ALT alanine aminotransferase, AST aspartate aminotransferase, CNS central nervous system, GI gastrointestinal, HER2 human epidermal growth factor receptor 2

a phase II trial conducted in Taiwan evaluating bevacizumab, etoposide, and cisplatin in breast cancer patients with progressive CNS after WBRT [22]. Our results are also in line with a phase II trial of patients with brain metastases from non-small cell lung cancer, where the combination of bevacizumab with carboplatin and paclitaxel achieved a CNS ORR of $61.2 \%$ [23]. Historical data with the combination of lapatinib plus capecitabine using identical response criteria have indicated a CNS response rate of $18-38 \%$ in patients with HER2-positive breast refractory cancer [16, 24-27]. The CNS response rate to lapatinib plus capecitabine was higher (67\%) in the upfront setting, but in a less heavily pre-treated population with no prior radiotherapy [28]. In our study, $87 \%$ of patients had progressed after radiotherapy, and $70 \%$ of HER2-positive patients had prior lapatinib. Furthermore, the CNS ORR we observed also compares favorably with that of neratinib and capecitabine (CNS ORR 49\%) and tucatinib-trastuzumab-capecitabine (CNS ORR 47\%) $[29,30]$. For patients with HER2-negative disease, options are substantially more limited. In TBCRC 018, the CNS ORR in patients with TNBC was $12 \%$ [31]. In the JPBO study, the CNS ORR in patient patients with ER+/HER2-negative disease was 5.8\% [32]. In addition, we observed a median OS of 14.10 months in the study overall, which compares favorably to historical data, where median survival in patients after progression through WBRT is 6 months or less [16, 33-35]. However, cross-trial comparisons must be interpreted with caution.

The rate of CNS hemorrhage seen in our study is consistent with the rates observed in prior reports [36]. These suggest that the use of bevacizumab-containing regimens is safe in patients with brain metastases from breast cancer. A similar low rate of CNS hemorrhage was seen with the use of bevacizumab in patients with brain metastases from non-small cell lung cancer [37].

Because bevacizumab can rapidly normalize vascular permeability and thus reduce gadolinium leakiness, one potential critique is that the high CNS response rate we

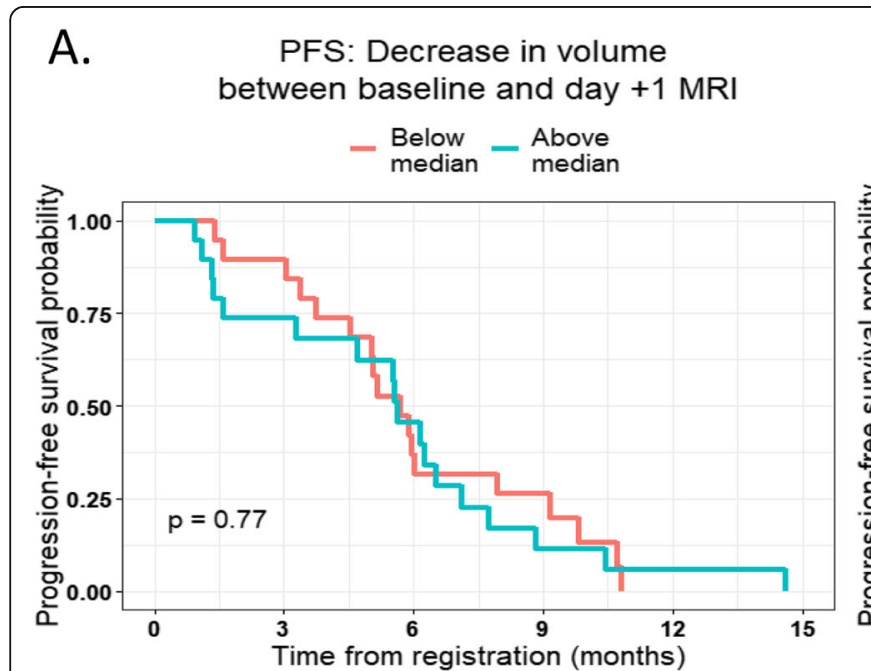

B. PFS: Percent decrease in volume between baseline and day $+1 \mathrm{MRI}$

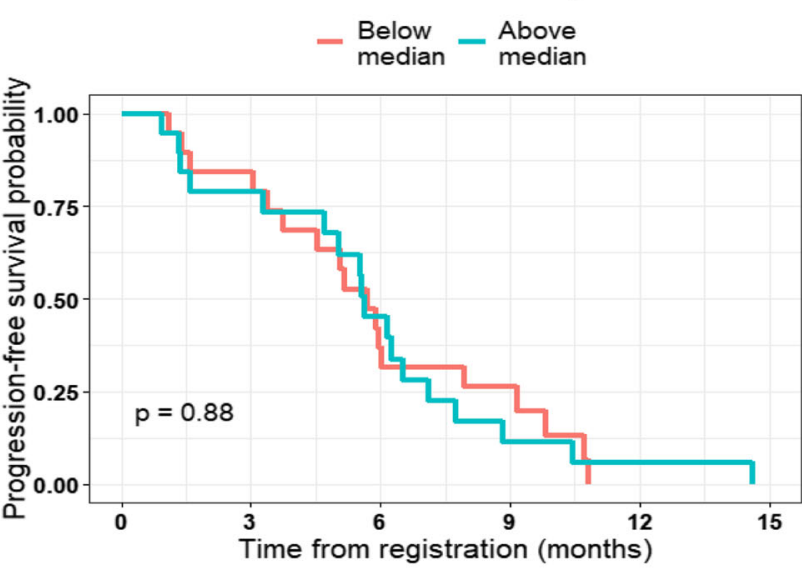

Number at risk (number of events)

\begin{tabular}{|c|ccc|c|c|}
\hline $19(0)$ & $17(2)$ & $7(12)$ & $4(14)$ & $0(18)$ & $0(18)$ \\
$19(0)$ & $13(5)$ & $8(10)$ & $2(16)$ & $1(17)$ & $0(18)$ \\
\hline 0 & 3 & 6 & 9 & 12 & 15 \\
\hline
\end{tabular}

Number at risk (number of events)
\begin{tabular}{|ccc|c|c|c|}
\hline $19(0)$ & $16(3)$ & $7(12)$ & $4(14)$ & $0(18)$ & $0(18)$ \\
\hline $19(0)$ & $14(4)$ & $8(10)$ & $2(16)$ & $1(17)$ & $0(18)$ \\
\hline 0 & 3 & 6 & 9 & 12 & 15 \\
\hline
\end{tabular}

Fig. 3 Association between progression-free survival (PFS) and $\mathbf{a}$ absolute decrease in volume between baseline MRI and day $+1 \mathrm{MRI}$, and $\mathbf{b}$ relative decrease in volume between baseline MRI and day +1 MRI 


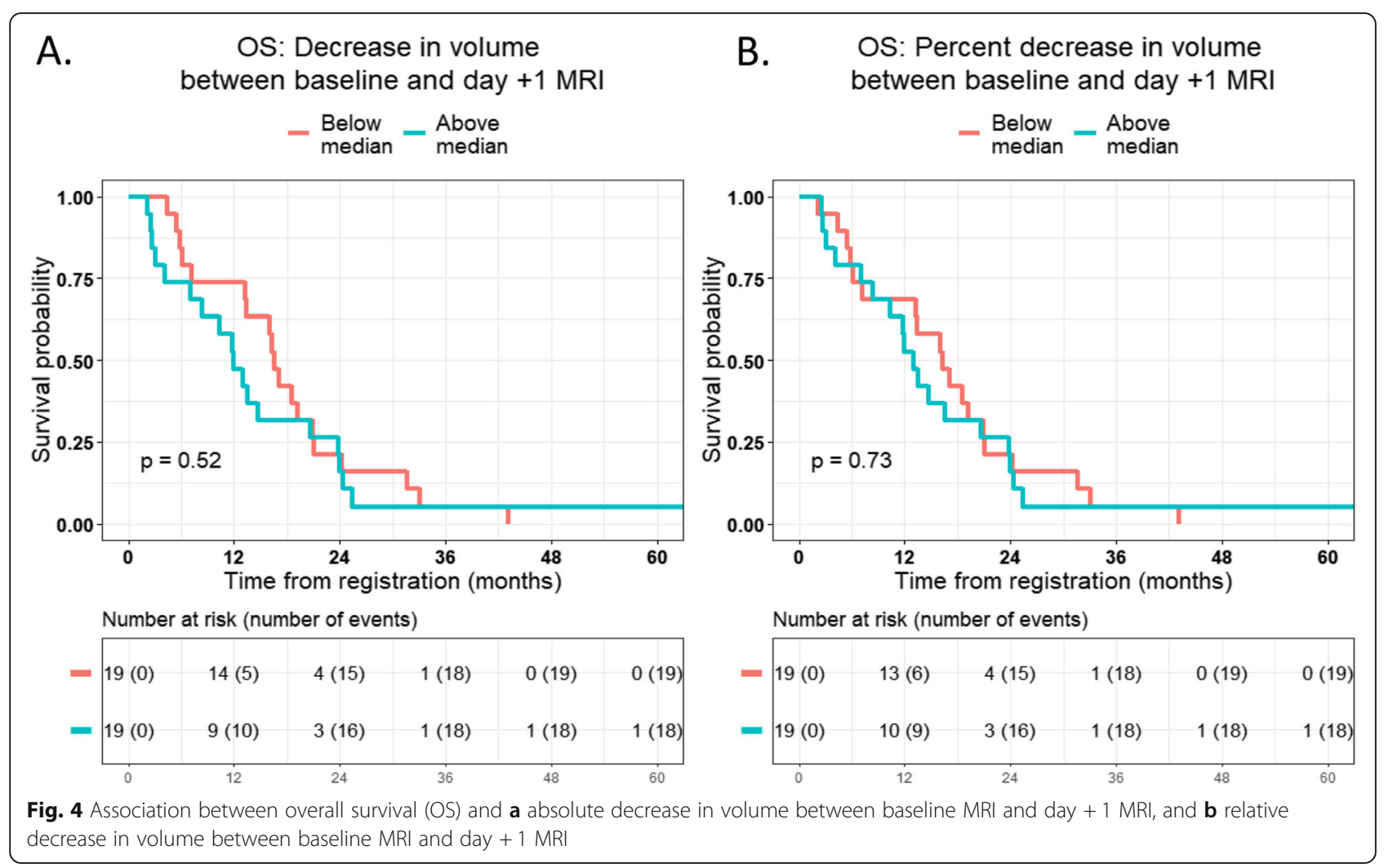

observed in our study could be related to purely radiological changes that do not necessarily confer clinical benefit $[38,39]$. However, only patients who also had simultaneous non-CNS disease stability or response were counted as CNS responders per our pre-specified definitions, supporting that patients were deriving clinical benefit. Finally, we did observe extracranial responses in a proportion of patients who had measurable extracranial metastases at study entry. While we did not identify a significant association between decrease in volume of CNS lesions and PFS or OS, these results should be interpreted with caution, as the number of patients for subgroup comparisons was small.

Our study had several limitations. First, we did not include bevacizumab-alone or carboplatin-alone arms and therefore cannot determine the individual contributions of these agents, although our efficacy results did exceed those of historical data with platinum alone $[6,7,9,40$, 41]. Second, we cannot exclude the possibility that trastuzumab may have contributed to the CNS response rate in patients with HER2-positive disease. Although we did observe responses in all major breast cancer subtypes, we are not able to formally compare outcomes by subtype. In addition, we did not include neurocognitive testing or patient-reported outcomes, which would have been a valuable addition in light of uncertainty regarding the meaning of radiographic responses in the setting of antiangiogenic therapies [42]. Similarly, presence or absence of edema was not quantified as part of this trial. However, the pre-specified response criteria used in the study provided a structured format for response evaluation. Finally, although we were not able to identify associations between VEGF genotypes and clinical outcomes, we acknowledge that our sample size was small, and our power limited.

\section{Conclusion}

We report a high rate of durable, objective CNS responses with the combination of bevacizumab with carboplatin in patients with breast cancer brain metastases, and that MRI changes detected as early as day +1 after a single dose of bevacizumab were associated with long-term clinical outcomes, including overall survival. Given the dearth of available treatment options for such patients, we believe that further study of bevacizumab-based regimens is warranted, possibly in combination with novel agents targeting key pathways in brain metastases.

\section{Supplementary information}

Supplementary information accompanies this paper at https://doi.org/10. 1186/s13058-020-01372-w.

Additional file 1. Supplementary material containing supplemental tables and figures.

\section{Abbreviations}

AE: Adverse event; ANC: Absolute neutrophil count; Cl: Confidence interval; CNS: Central nervous system; ECOG PS: Eastern Cooperative Oncology Group 
performance status; ER: Estrogen receptor; HER2: Human epidermal growth factor receptor 2; HR: Hazard ratio; LVEF: Left ventricular ejection fraction; ORR: Overall response rate; OS: Overall survival; PFS: Progression-free survival; RANO-BM: Response assessment in neuro-oncology brain metastases: RT: Radiation therapy; SRS: Stereotactic radiosurgery; VEGF: Vascular endothelial growth factor; WBRT: Whole breast radiation therapy

\section{Acknowledgements}

We want to thank Elizabeth Bullitt from University of North Carolina at Chapel Hill, Chapel Hill, NC, for her help with this study. This study was previously presented in part at the 49th Annual American Society of Clinical Oncology Meeting, Chicago, IL, May 31-June 4, 2013.

\section{Clinical trial registration}

The study is registered at www.clinicaltrials.gov (NCT01004172).

\section{Authors' contributions}

NUL, RSG, BPS, AGS, GH, IEK, and EPW contributed to the conception or design of the work. All authors contributed to the acquisition, analysis, or interpretation of data for the work. All authors contributed to the drafting or revising the manuscript text. All authors gave final approval of the version submitted.

\section{Funding}

The American Society of Clinical Oncology Career Development Award, the Breast Cancer Research Foundation (to N.U.L. and E.P.W.), the Dana-Farber Cancer Institute Women's Cancers Program Executive Council Personalized Medicine Award, the South-Eastern Norway Regional Health Authority Grants 2016102 and 2013069 (to K.E.E.), the Norwegian Cancer Society Grant 6817564 (to K.E.E.), and Genentech gave funding. None of the funders had a role in the design of the study, the collection, analysis, and interpretation of data and in writing the manuscript.

\section{Availability of data and materials}

The datasets used and/or analyzed during the current study are available from the corresponding author on reasonable request.

\section{Ethics approval and consent to participate}

The protocol was approved by the Dana-Farber/Harvard Cancer Center (DF/ HCC) Scientific Review Committee and Institutional Review Board. All study participants provided written informed consent prior to any study-related procedures.

\section{Consent for publication}

Not applicable.

\section{Competing interests}

Harris: Member, Novometrics LLC, IQ Medical Imaging LLC; Scientific Advisory Board, Fovia Inc.

Krop: Institutional research funding from Genentech/Roche, Pfizer, and Daiichi-Sankyo; advisory board participant/consultant for Genentech/Roche, Daiichi-Sankyo, Macrogenics, Context Therapeutics, Taiho Oncology, BristolMyers Squibb, Celltrion Pharma, Merck (DSMC), Novartis (DSMB); honoraria from Genentech/Roche, Daiichi-Sankyo, Macrogenics, Context Therapeutics, Taiho.

Morganstern: advisory role and honoraria for PUMA pharmaceuticals. Winer: Institutional research funding from Genentech/Roche; consultant/ honoraria from Genentech/Roche, GSK and Seattle Genetics (each < \$5000). Lin: Consulting for Puma, Seattle Genetics, Daichii Sankyo though none would have a financial interest in the results or materials of this study.

\section{Author details}

'Dana-Farber Cancer Institute, Dana-Farber/Brigham \& Women's Cancer Center, 450 Brookline Avenue, Boston, MA 02215, USA. ²Department of Diagnostic Physics, Oslo University Hospital, Oslo, Norway. Indiana University School of Medicine, Indianapolis, IN, USA. ${ }^{4}$ Massachusetts General Hospital, Boston, MA, USA. ${ }^{5}$ University of Texas Southwestern Medical Center, Dallas, TX, USA. 'IMRIS Inc., Minnetonka, MN, USA.
Received: 29 July 2020 Accepted: 15 November 2020

Published online: 30 November 2020

\section{References}

1. Lin NU, Amiri-Kordestani L, Palmieri D, Liewehr DJ, Steeg PS. CNS metastases in breast cancer: old challenge, new frontiers. Clin Cancer Res. 2013;19(23):6404-18

2. Pestalozzi BC, Holmes E, de Azambuja E, Metzger-Filho O, Hogge L, Scullion $M$, et al. CNS relapses in patients with HER2-positive early breast cancer who have and have not received adjuvant trastuzumab: a retrospective substudy of the HERA trial (BIG 1-01). Lancet Oncol. 2013;14(3):244-8.

3. Bullitt E, Lin NU, Smith JK, Zeng D, Winer EP, Carey LA, et al. Blood vessel morphologic changes depicted with MR angiography during treatment of brain metastases: a feasibility study. Radiology. 2007;245(3):824-30.

4. Kim LS, Huang S, Lu W, Lev DC, Price JE. Vascular endothelial growth factor expression promotes the growth of breast cancer brain metastases in nude mice. Clin Exp Metastasis. 2004;21 (2):107-18.

5. Kodack DP, Chung E, Yamashita H, Incio J, Duyverman AM, Song Y, et al. Combined targeting of HER2 and VEGFR2 for effective treatment of HER2amplified breast cancer brain metastases. Proc Natl Acad Sci U S A. 2012; 109(45):E3119-27.

6. Miller K, Wang M, Gralow J, Dickler M, Cobleigh M, Perez EA, et al. Paclitaxel plus bevacizumab versus paclitaxel alone for metastatic breast cancer. N Engl J Med. 2007;357(26):2666-76.

7. Pegram M, Chan D, Dichmann RA, Tan-Chiu E, Yeon C, Durna L, et al. Phase Il combined biological therapy targeting the HER-2 proto-oncogene and the vascular endothelial growth factor using trastuzumab (T) and Avastin (B) as first-line treatment of HER2-amplified breast cancer. Breast Cancer Res Treat. 2006;100(Suppl 1):asbstr 301.

8. Stewart DJ, Mikhael NZ, Nair RC, Kacew S, Montpetit V, Nanji A, et al. Platinum concentrations in human autopsy tumor samples. Am J Clin Oncol. 1988;11(2):152-8.

9. Franciosi V, Cocconi G, Michiara M, Di Costanzo F, Fosser V, Tonato M, et al. Front-line chemotherapy with cisplatin and etoposide for patients with brain metastases from breast carcinoma, nonsmall cell lung carcinoma, or malignant melanoma: a prospective study. Cancer. 1999;85(7):1599-605.

10. Jahnke K, Muldoon LL, Varallyay CG, Lewin SJ, Kraemer DF, Neuwelt EA. Bevacizumab and carboplatin increase survival and asymptomatic tumor volume in a glioma model. Neuro-Oncology. 2009;11(2):142-50.

11. Arteaga CL, Mayer IA, O'Neill AM, Swaby RF, Alpaugh RK, Yang XJ, et al. A randomized phase III double-blinded placebo-controlled trial of first-line chemotherapy and trastuzumab with or without bevacizumab for patients with HER2/neu-overexpressing metastatic breast cancer (HER2+ MBC): A trial of the Eastern Cooperative Oncology Group (E1 105). J Clin Oncol. 2012; 30:abstract 605.

12. Lambrechts D, Lenz HJ, de Haas S, Carmeliet P, Scherer SJ. Markers of response for the antiangiogenic agent bevacizumab. J Clin Oncol. 2013; 31(9):1219-30.

13. Miles DW, Dieras V, Cortes J, Duenne AA, Yi J, O'Shaughnessy J. First-line bevacizumab in combination with chemotherapy for HER2-negative metastatic breast cancer: pooled and subgroup analyses of data from 2447 patients. Ann Oncol. 2013;24(11):2773-80.

14. Gianni L, Romieu GH, Lichinitser M, Serrano SV, Mansutti M, Pivot X, et al. AVEREL: a randomized phase III trial evaluating bevacizumab in combination with docetaxel and trastuzumab as first-line therapy for HER2positive locally recurrent/metastatic breast cancer. J Clin Oncol. 2013;31(14): $1719-25$.

15. Lin NU, Seah DS, Gelman R, Desantis S, Mayer EL, Isakoff S, et al. A phase II study of bevacizumab in combination with vinorelbine and trastuzumab in HER2-positive metastatic breast cancer. Breast Cancer Res Treat. 2013;139(2): 403-10.

16. Lin NU, Dieras V, Paul D, Lossignol D, Christodoulou C, Stemmler HJ, et al. Multicenter phase II study of lapatinib in patients with brain metastases from HER2-positive breast cancer. Clin Cancer Res. 2009;15(4):1452-9.

17. Sorensen AG, Patel S, Harmath C, Bridges S, Synnott J, Sievers A, et al. Comparison of diameter and perimeter methods for tumor volume calculation. J Clin Oncol. 2001;19(2):551-7.

18. Lin NU, Lee EQ, Aoyama H, Barani IJ, Barboriak DP, Baumert BG, et al. Response assessment criteria for brain metastases: proposal from the RANO group. Lancet Oncol. 2015;16(6):e270-8. 
19. Robert NJ, Dieras V, Glaspy J, Brufsky A, Bondarenko I, Lipatov O, et al. RIBBON-1: Randomized, double-blind, placebo-controlled, phase III trial of chemotherapy with or without bevacizumab (B) for first-line treatment of HER2-negative locally recurrent or metastatic breast cancer (MBC). J Clin Oncol. 2009;27(15s):abstr 1005.

20. Miles D, Chan A, Romieu G, Dirix LY, Cortes J, Pivot X, et al. Randomized, double-blind, placebo-controlled, phase III study of bevacizumab with docetaxel or docetaxel with placebo as first-line therapy for patients with locally recurrent or metastatic breast cancer (mBC): AVADO. J Clin Oncol. 2008;26(May 20 suppl):abstr LBA1011.

21. Pivot $X$, Schneeweiss A, Verma S, Thomssen C, Passos-Coelho JL, Benedetti $\mathrm{G}$, et al. Efficacy and safety of bevacizumab in combination with docetaxel for the first-line treatment of elderly patients with locally recurrent or metastatic breast cancer: results from AVADO. Eur J Cancer. 2011:47(16): 2387-95.

22. Lu YS, Chen TW, Lin CH, Yeh DC, Tseng LM, Wu PF, et al. Bevacizumab preconditioning followed by etoposide and cisplatin is highly effective in treating brain metastases of breast cancer progressing from whole-brain radiotherapy. Clin Cancer Res. 2015:21(8):1851-8.

23. Besse B, Le Moulec S, Mazieres J, Senellart H, Barlesi F, Chouaid C, et al. Bevacizumab in patients with nonsquamous non-small cell lung cancer and asymptomatic, untreated brain metastases (BRAIN): a nonrandomized, phase II study. Clin Cancer Res. 2015;21(8):1896-903.

24. Metro G, Foglietta J, Russillo M, Stocchi L, Vidiri A, Giannarelli D, et al. Clinical outcome of patients with brain metastases from HER2-positive breast cancer treated with lapatinib and capecitabine. Ann Oncol. 2010; 22(3):625-30.

25. Boccardo F, Kaufman B, Baselga J, Dieras V, Link J, Casey MA, et al Evaluation of lapatinib (Lap) plus capecitabine (Cap) in patients with brain metastses (BM) from HER2+ breast cancer (BC) enrolled in the Lapatinib Expanded Access Program (LEAP) and French Authorisation Temporaire d'Utilisation (ATU). J Clin Oncol. 2008;26:abstract 1094.

26. Sutherland S, Ashley S, Miles D, Chan S, Wardley A, Davidson N, et al. Treatment of HER2-positive metastatic breast cancer with lapatinib and capecitabine in the lapatinib expanded access programme, including efficacy in brain metastases--the UK experience. Br J Cancer. 2010;102(6): 995-1002.

27. Lin NU, Eierman W, Greil R, Campone M, Kaufman B, Steplewski K, et al. Randomized phase II study of lapatinib plus capecitabine or lapatinib plus topotecan for patients with HER2-positive breast cancer brain metastases. J Neuro-Oncol. 2011;105(3):613-20.

28. Bachelot T, Romieu CG, Campone M, Dieras V, Cropet C, Roche HH, et al. LANDSCAPE: An FNCLCC phase II study with lapatinib $(L)$ and capecitabine (C) in patients with brain metastases (BM) from HER2-positive (+) metastatic breast cancer (MBC) before whole-brain radiotherapy (WBR). J Clin Oncol. 2011;29(suppl):abstr 209.

29. Freedman RA, Gelman RS, Anders CK, Melisko ME, Parsons HA, Cropp AM, et al. TBCRC 022: a phase II trial of neratinib and capecitabine for patients with human epidermal growth factor receptor 2-positive breast cancer and brain metastases. J Clin Oncol. 2019;37(13):1081-9.

30. Lin NU, Murthy R, Anders C, Borges V, Hurvitz S, Loi S, et al. Tucatinib vs placebo added to trastuzumab and capecitabine for patients with previously treated HER2+ metastatic breast cancer with brain metastases (HER2CLIMB) [abstract]. ASCO Annual Meeting; May 29-June 2, 2020; Chicago, IL.

31. Anders C, Deal AM, Abramson V, Liu MC, Storniolo AM, Carpenter JT, et al. TBCRC 018: phase II study of iniparib in combination with irinotecan to treat progressive triple negative breast cancer brain metastases. Breast Cancer Res Treat. 2014;146(3):557-66.

32. Anders CK, Le Rhun E, Bachelot TD, Yardley DA, Awada A, Conte PF, et al. A phase II study of abemaciclib in patients (pts) with brain metastases (BM) secondary to HR+, HER2-metastatic breast cancer (MBC). J Clin Oncol. 2019; https://doi.org/10.1200/JCO.2019.37.15_suppl.1017.

33. Lin NU, Claus E, Sohl J, Razzak AR, Arnaout A, Winer EP. Sites of distant recurrence and clinical outcomes in patients with metastatic triple-negative breast cancer: high incidence of central nervous system metastases. Cancer. 2008;113(10):2638-45.

34. Dawood S, Broglio K, Esteva FJ, Yang W, Kau SW, Islam R, et al. Survival among women with triple receptor-negative breast cancer and brain metastases. Ann Oncol. 2009;20(4):621-7.
35. Anders CK, Deal AM, Miller CR, Khorram C, Meng H, Burrows E, et al. The prognostic contribution of clinical breast cancer subtype, age, and race among patients with breast cancer brain metastases. Cancer. 2011;117(8): 1602-11.

36. Besse B, Lasserre SF, Compton P, Huang J, Augustus S, Rohr UP. Bevacizumab safety in patients with central nervous system metastases. Clin Cancer Res. 2010;16(1):269-78.

37. Socinski MA, Langer CJ, Huang JE, Kolb MM, Compton P, Wang L, et al. Safety of bevacizumab in patients with non-small-cell lung cancer and brain metastases. J Clin Oncol. 2009;27(31):5255-61.

38. Wen PY, Macdonald DR, Reardon DA, Cloughesy TF, Sorensen AG, Galanis E, et al. Updated response assessment criteria for high-grade gliomas: response assessment in neuro-oncology working group. J Clin Oncol. 2010; 28(11):1963-72.

39. Batchelor TT, Sorensen AG, di Tomaso E, Zhang WT, Duda DG, Cohen KS, et al. AZD2171, a pan-VEGF receptor tyrosine kinase inhibitor, normalizes tumor vasculature and alleviates edema in glioblastoma patients. Cancer Cell. 2007;11(1):83-95.

40. Perez EA. Carboplatin in combination therapy for metastatic breast cancer. Oncologist. 2004:9(5):518-27.

41. Gradishar WJ, Stephenson P, Glover DJ, Neuberg DS, Moore MR, Windschitl HE, et al. A phase II trial of cisplatin plus WR-2721 (amifostine) for metastatic breast carcinoma: an Eastern Cooperative Oncology Group Study (E8188). Cancer. 2001;92(10):2517-22.

42. Wefel JS, Cloughesy T, Zazzali JL, Zheng M, Prados M, Wen PY, et al. Neurocognitive function in patients with recurrent glioblastoma treated with bevacizumab. Neuro-Oncology. 2011;13(6):660-8.

\section{Publisher's Note}

Springer Nature remains neutral with regard to jurisdictional claims in published maps and institutional affiliations.

Ready to submit your research? Choose BMC and benefit from:

- fast, convenient online submission

- thorough peer review by experienced researchers in your field

- rapid publication on acceptance

- support for research data, including large and complex data types

- gold Open Access which fosters wider collaboration and increased citations

- maximum visibility for your research: over $100 \mathrm{M}$ website views per year

At $\mathrm{BMC}$, research is always in progress.

Learn more biomedcentral.com/submissions 